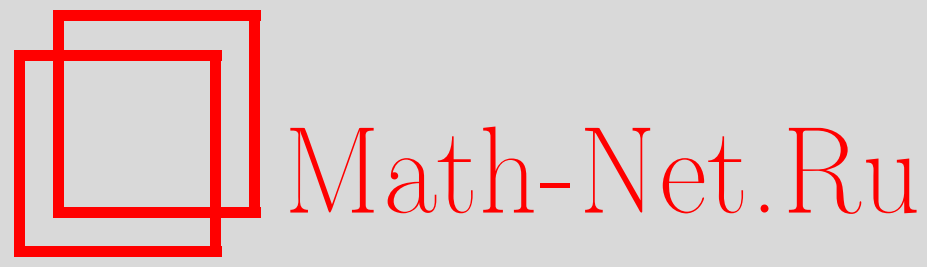

Е. А. Македонский, О диких и ручных конечномерных алгебрах Ли, Функи. анализ и его прил., 2013, том 47, выпуск 4, 30-44

DOI: https://doi.org/10.4213/faa3130

Использование Общероссийского математического портала MathNet.Ru подразумевает, что вы прочитали и согласны с пользовательским соглашением

http://www . mathnet.ru/rus/agreement

Параметры загрузки:

IP : 35.174 .16 .151

26 апреля 2023 г., 14:05:44

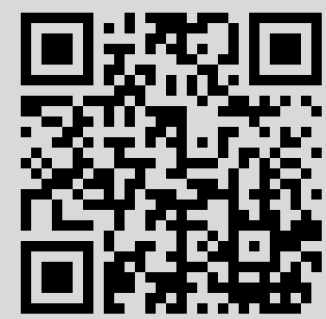


Функционалъный анализ и его приложения

2013, т. 47, вып. 4, с. 30-44

УДК $512.554+552.552$

\title{
О диких и ручных конечномерных алгебрах Ли
}

\author{
(c) 2013. Е. А. МАКЕДОНСКИЙ
}

Найдены все конечномерные алгебры Ли над алгебраически замкнутым полем нулевой характеристики, для которых задача классификации конечномерных представлений не является дикой.

\section{§1. Введение}

Все рассматриваемые алгебры определены над фиксированным алгебраически замкнутым полем $\mathbb{K}$ нулевой характеристики и конечномерны над $\mathbb{K}$. В дальнейшем поле мы часто указывать не будем; в частности, вместо $s l_{n}(\mathbb{K})$ будем писать просто $s l_{n}$.

В настоящей работе рассматривается следующая задача: для данной конечномерной алгебры Ли $L$ классифицировать все ее неразложимые конечномерные представления. В случае, когда алгебра Ли $L$ полупроста, ответ известен с начала двадцатого века: все представления такой алгебры классифицируются некоторыми элементами решетки весов. Мы будем исследовать вопрос о возможности подобной классификации для других конечномерных алгебр Ли.

Под невозможностью классификации представлений мы будем понимать дикость (см. определения 1, 2), введенную Ю. А. Дроздом в работе [2]. Неформально дикость классификационной задачи означает, что из ее решения будет следовать решение задачи классификации пары матриц с точностью до подобия (или, что то же самое, классификации всех конечномерных представлений свободной ассоциативной алгебры с двумя образующими). Эта задача считается чрезвычайно сложной, так как ее решение включает в себя решения задач классификации всех конечномерных представлений всех конечномерных ассоциативных алгебр. Для некоторого класса задач, включающего классификацию представлений конечномерных ассоциативных алгебр, Ю. А. Дрозд показал, что эти задачи разбиваются на два класса - диких и «ручных», когда множество всех представлений распадается на конечное множество однопараметрических семейств. Для получения аналогичного результата для конечномерных алгебр Ли нам потребуется слегка изменить определение ручных задач (см. определение 3 ).

Имеется много работ (см., например, [1]-[3], [5], [6]), в которых в некоторых классах задач теории представлений выделены ручные и дикие задачи.

Данная работа посвящена вопросу о том, для каких алгебр Ли $L$ задача классификации конечномерных представлений является дикой или ручной. Такие алгебры Ли будем называть соответственно дикими и ручными. Основной результат данной работы:

Теорема 3. Ручными являются следуюшие алгебры Ли:

(1) nолупростые;

(2) прямые суммы полупростой и одномерной.

Все остальные алгебры Ли дикие. 
Таким образом, конечномерные алгебры Ли разбиваются на два класса ручных и диких.

В данной работе представления неполупростых алгебр Ли изучаются при помощи некоторого бесконечного колчана. Алгебра путей этого колчана не изоморфна обертывающей алгебре исходной алгебры Ли, потому что в ней бесконечно много идемпотентов. Однако категория представлений этой алгебры эквивалентна категории представлений исходной алгебры Ли.

О структуре работы. В $\S 2$ приведены определения дикости и ручности и некоторые простые примеры ручных и диких алгебр Ли. В $\S 3$ изучается задача описания представлений алгебр Ли с абелевым радикалом, а именно, она сводится к задаче описания представлений некоторого колчана с соотношениями, а в $\S 4$ с помощью исследования представлений этого колчана алгебры Ли с абелевым радикалом разделены на ручные и дикие. Далее, после исследования алгебр Ли с ручным фактором по коммутанту радикала, в §5 доказывается основная теорема.

\section{§2. Примеры ручных и диких алгебр Ли}

Пусть $\mathbb{K}\langle x, y\rangle$ - свободная ассоциативная алгебра с двумя образующими.

Определение 1 [2]. Ассоциативная алгебра $A$ называется дикой, если существует такой $A$ - $\mathbb{K}\langle x, y\rangle$-бимодуль $M$, являющийся свободным модулем конечного ранга над $\mathbb{K}\langle x, y\rangle$, что функтор $M \otimes_{\mathbb{K}\langle x, y\rangle}(-): \bmod (\mathbb{K}\langle x, y\rangle) \rightarrow \bmod (A)$ сохраняет неразложимость и переводит неизоморфные модули в неизоморфные.

Это определение дикости было исторически первым. Мы будем пользоваться несколько другим определением дикости, эквивалентным приведенному в работе [4]. Оно имеет смысл для любой алгебры, у которой определена категория конечномерных представлений. Мы будем применять его для алгебр Ли.

Определение 2 [4]. Алгебра $A$ называется дикой, если существует точный функтор из категории представлений алгебры $\mathbb{K}\langle x, y\rangle$ в категорию представлений алгебры $A$, сохраняющий неразложимость и переводящий неизоморфные модули в неизоморфные.

Два рассматриваемых определения эквивалентны как минимум для конечномерных ассоциативных алгебр. Кроме того, из дикости в смысле определения 1 следует дикость в смысле определения 2.

Определение 3. Алгебра $A$ называется ручной, если для каждого натурального $n$ множество всех ее неразложимых представлений размерности $n$ распадается на конечное множество однопараметрических семейств.

Это определение мы будем применять и для алгебр Ли.

Исследованию связи понятий дикости и ручности для конечномерных ассоциативных алгебр посвящена работа [2], в которой доказано, что любая такая алгебра является либо дикой, либо ручной.

В рамках второго определения дикости и определения ручности очевидно, что алгебры с эквивалентными категориями конечномерных представлений дикими или ручными могут быть только одновременно.

Следующее простое предложение мы будем использовать, не ссылаясь на него, на протяжении всей работы.

Предложение 1. Пусть $I-$ - деал алгебры A. Если алгебра $A / I$ дикал, то и алгебра А дикал. 
Доказательство. Допустим, что существует точный функтор из категории представлений алгебры $\mathbb{K}\langle x, y\rangle$ в категорию представлений алгебры $A / I$, удовлетворяющий условиям определения 2 . Рассматривая $A / I$-модули как $A$-модули, мы получаем тогда функтор в категорию представлений алгебры $A$, также удовлетворяющий условиям определения 2 .

Приведем некоторые примеры ручных и диких алгебр Ли.

2.1. Двумерные алгебры Ли дикие. Пусть $L$ - двумерная алгебра Ли. Тогда она либо абелева, либо имеет такой базис $\{a, b\}$, что $[a, b]=b$. Во втором случае алгебра $L$ является дикой (см., например, $[6])$. Представления двумерной абелевой алгебры Ли $L=\langle a, b\rangle$ - это представления алгебры полиномов от двух переменных, дикость которой является хорошо известным фактом [1]. Тем не менее для иллюстрации приведем доказательство (см., например, [6]).

Рассмотрим $L$ - $\mathbb{K}\langle x, y\rangle$-бимодуль $M$, являющийся свободным $\mathbb{K}\langle x, y\rangle$-модулем ранга 4. Образующие $a$ и $b$ алгебры $L$ действуют на этом бимодуле следующими $\mathbb{K}\langle x, y\rangle$-линейными преобразованиями:

$$
a=\left(\begin{array}{cccc}
0 & 0 & 1 & 0 \\
0 & 0 & 0 & x \\
0 & 0 & 0 & 1 \\
0 & 0 & 0 & 0
\end{array}\right), \quad b=\left(\begin{array}{cccc}
0 & 1 & 0 & 0 \\
0 & 0 & 0 & y \\
0 & 0 & 0 & x \\
0 & 0 & 0 & 0
\end{array}\right) .
$$

Нетрудно видеть, что эти преобразования коммутируют, поэтому это действительно $L$ - $\mathbb{K}\langle x, y\rangle$-бимодуль. Рассмотрим конечномерный неразложимый $\mathbb{K}\langle x, y\rangle$ модуль $V$. Пусть $x$ и $y$ действуют на этом модуле с помощью матриц $X$ и $Y$. Тогда $N=M \otimes_{\mathbb{K}\langle x, y\rangle} V$ является векторным пространством, изоморфным $V \oplus$ $V \oplus V \oplus V$, на котором образующие рассматриваемой алгебры Ли действуют при помощи следующих операторов:

$$
a=\left(\begin{array}{cccc}
0 & 0 & 1 & 0 \\
0 & 0 & 0 & X \\
0 & 0 & 0 & 1 \\
0 & 0 & 0 & 0
\end{array}\right), \quad b=\left(\begin{array}{cccc}
0 & 1 & 0 & 0 \\
0 & 0 & 0 & Y \\
0 & 0 & 0 & X \\
0 & 0 & 0 & 0
\end{array}\right) .
$$

Допустим, что этот модуль разложим. Пусть $N=W_{1} \oplus W_{2}$ - прямая сумма $L$-модулей. Положим $V_{i}=\left\{u_{4} \mid\left(u_{1}, u_{2}, u_{3}, u_{4}\right)^{t} \in W_{i}\right\}$ для некоторых $u_{1}, u_{2}$, $u_{3}, i=1,2$. Очевидно, что $V=V_{1}+V_{2}$. Пусть $\left(u_{1}, u_{2}, u_{3}, u_{4}\right)^{t} \in W_{i}$. Применив к этому элементу оператор $a^{2}$, мы получим элемент $\left(u_{4}, 0,0,0\right)^{t} \in W_{i}$. Значит, $\left(V_{i}, 0,0,0\right) \in W_{i}$. Поэтому $V_{1} \cap V_{2}=\{0\}$ и $V=V_{1} \oplus V_{2}, W_{i} \cap(V, 0,0,0)^{t}=$ $\left(V_{i}, 0,0,0\right)^{t}$. Далее, применим к элементу $\left(u_{1}, u_{2}, u_{3}, u_{4}\right)^{t} \in W_{i}$ операторы $a b$ и $b^{2}$. Получим элементы $\left(x u_{4}, 0,0,0\right)^{t} \in W_{i}$ и $\left(y u_{4}, 0,0,0\right)^{t} \in W_{i}$. Поэтому $x V_{i} \in V_{1}, y V_{i} \in V_{i}, i=1,2$. Мы получили разложение исходного представления алгебры $\mathbb{K}\langle x, y\rangle$. Значит, построенный функтор переводит неразложимые представления в неразложимые.

Пусть теперь для двух представлений $V_{1}, V_{2}$ алгебры $\mathbb{K}\langle x, y\rangle$ представления $N_{1}=M \otimes_{\mathbb{K}\langle x, y\rangle} V_{1}, N_{2}=M \otimes_{\mathbb{K}\langle x, y\rangle} V_{2}$ изоморфны. Тогда их размерности равны и существует обратимый сплетающий оператор, т. е. такой оператор $S$, что $S a_{1}=a_{2} S, S b_{1}=b_{2} S$ (номер у операторов $a_{i}, b_{i}$ соответствует номеру представления $\left.V_{i}\right)$. Запишем $S$ в виде 


$$
S=\left(\begin{array}{llll}
S_{11} & S_{12} & S_{13} & S_{14} \\
S_{21} & S_{22} & S_{23} & S_{24} \\
S_{31} & S_{32} & S_{33} & S_{34} \\
S_{41} & S_{42} & S_{43} & S_{44}
\end{array}\right)
$$

Теперь, перемножив блочные матрицы и приравняв коэффициенты, мы получим, что $S_{21}=S_{31}=S_{41}=S_{32}=S_{42}=S_{43}=S_{23}=0, S_{11}=S_{22}=S_{33}=S_{44}$. Кроме того, $S_{22} Y_{1}=Y_{2} S_{44}, S_{22} X_{1}=X_{2} S_{44}$. Поэтому мы получили сплетающий оператор для представлений $V_{1}, V_{2}$. Он обратим, так как в силу равенства нулю всех блоков ниже диагонали в блочной матрице $S$ и равенства всех ее диагональных блоков $\operatorname{det} S=\operatorname{det} S_{22}^{4}$, т. е. матрица $S_{22}$ обратима. Значит, описываемый функтор переводит неизоморфные представления в неизоморфные. Этот факт завершает доказательство дикости двумерной абелевой алгебры Ли.

Следовательно, все двумерные алгебры Ли дикие.

2.2. Разрешимые алгебры Ли дикие. Пусть теперь $I$ - идеал алгебры Ли $L, L \triangleright I$, и $\operatorname{dim}(L / I)=2$. Тогда $L$ дикая, так как $L / I$ двумерна, а следовательно, дикая.

В частности, если $L$ - разрешимая алгебра Ли, то в силу теоремы Ли в ней есть флаг из идеалов, в частности, идеал любой размерности, не превышающей размерности самой алгебры. Следовательно, если размерность алгебры $L$ не меньше двух, то в ней существует идеал коразмерности 2. Таким образом, верно следующее утверждение:

Предложение 2. Все разрешимые алгебры Ли размерности не менъше двух являются дикими.

2.3. Полупростые алгебры Ли ручные. Из классической теории представлений полупростых алгебр Ли известно, что неприводимые представления такой алгебры параметризуются доминантными целочисленными линейными формами на корневой решетке и исчерпывают все ее неразложимые представления. Поэтому все полупростые алгебры Ли ручные.

2.4. Одномерные расширения полупростых алгебр Ли ручные. Следующее утверждение должно быть известно, но автору не удалось найти подходящую ссылку, поэтому оно здесь приведено с полным доказательством. Это доказательство аналогично доказательству из книги [7, с. 225].

Предложение 3. Пусть $L=\widehat{L} \oplus L_{1}$, где $\widehat{L}-$ полупростая алгебра Ли, u $(M, f)$ - неразложимое представление алгебры Ли L. Тогда существуют такие неразложсимые представления $\left(M_{1}, f_{1}\right)$ u $\left(M_{2}, f_{2}\right)$ алгебр $\widehat{L}$ u $L_{1}$ соответственно, что $M=M_{1} \otimes M_{2} u f(X+Y)=f_{1}(X) \otimes \mathrm{id}+\mathrm{id} \otimes f_{2}(Y)$, где $X \in \widehat{L}$, $Y \in L_{1}$, id - тождественный оператор.

Доказательство. Поскольку ограничение представления $f$ на $\widehat{L}$ вполне приводимо (в силу полупростоты алгебры $\widehat{L}$ ), мы можем считать, что

$$
f(X)=\left(\begin{array}{cccc}
F_{1}(X) & 0 & \ldots & 0 \\
0 & F_{2}(X) & \ldots & 0 \\
\vdots & \vdots & \ddots & \vdots \\
0 & 0 & \ldots & F_{k}(X)
\end{array}\right)
$$


где $F_{i}$ - неприводимое представление алгебры $\widehat{L}$ размерности $h_{i}$. Далее можно считать, что $F_{1}=\cdots=F_{p}$, а представления $F_{q}, q>p$, не эквивалентны представлению $F_{1}$. Пусть $S_{i j}$ суть $\left(h_{i} \times h_{j}\right)$-матрицы и

$$
S=\left(\begin{array}{ccc}
S_{11} & \ldots & S_{1 k} \\
\vdots & \ddots & \vdots \\
S_{k 1} & \ldots & S_{k k}
\end{array}\right) \in \operatorname{gl}(n, \mathbb{K})
$$

Предположим, что $f(X) S=S f(X)$ для любого $X \in \widehat{L}$. Тогда $f_{i}(X) S_{i j}=$ $S_{i j} f_{j}(X)$ при $i, j=1, \ldots, k$. По лемме Шура [7, с. 225] $S_{i j}=s_{i j} 1_{h_{1}}, s_{i j} \in \mathbb{K}$, при $i, j=1, \ldots, p$ и $S_{i j}=0$ при $i \leqslant p<j$ и $j \leqslant p<i$.

Теперь применим этот результат к $S=f(Y), Y \in L_{1}$. Если бы $p \neq k$, то из соотношений $S_{i j}=0$ при $i \leqslant p<j$ и $j \leqslant p<i$ следовало бы, что представление $(M, f)$ разложимо. Значит, $p=k$.

Положим $f_{1}(X)=F_{1}(X)$ для $X \in \widehat{L}, f_{2}(Y)=\left(s_{i j}\right) \in g l(p, \mathbb{K}), Y \in L_{1}$. Пространство $M$ очевидным образом распадается в тензорное произведение, причем

$$
f(X)=f_{1}(X) \otimes 1_{p}, \quad X \in \widehat{L}, \quad f(Y)=1_{h} \otimes f_{2}(Y), \quad Y \in L_{1} .
$$

Очевидно, что если представление $\left(M_{2}, f_{2}\right)$ распадается в прямую сумму двух представлений, то и представление $f$ также распадается в прямую сумму двух представлений. Следовательно, $\left(M_{2}, f_{2}\right)$ - неразложимое представление. Обратно, если $\left(M_{2}, f_{2}\right)$ неразложимо, то и представление $(M, f)$ также неразложимо.

Замечание 1. Из предыдущего следует, что прямая сумма ручной и полупростой алгебр Ли ручная. Представления одномерной алгебры Ли $\langle e\rangle$, очевидно, задаются образом элемента $e$. Следовательно, неразложимое представление алгебры Ли $L=\widehat{L} \oplus\langle e\rangle$, где $\widehat{L}$ - полупростая алгебра Ли, задается неприводимым представлением алгебры $\widehat{L}$ и жордановой клеткой. Поэтому все такие алгебры Ли ручные.

Замечание 2. Если один из сомножителей приводим, то и тензорное произведение приводимо. Поэтому неприводимые представления алгебры Ли $L=$ $\widehat{L} \oplus\langle e\rangle$, где $\widehat{L}$ - полупростая алгебра Ли, - это неприводимые представления алгебры $\widehat{L}$, на которых элемент $е$ действует умножением на константу.

\section{§3. Колчан алгебры Ли с абелевым радикалом}

Сведем теперь исследование представлений алгебры Ли с абелевым радикалом к исследованию представлений некоторого колчана.

Лемма 1. Пусть $\widehat{L}=L \curlywedge I-$ алгебра Ли, такая, что $L$ полупроста, $u$ $I$ - абелев идеал. Тогда категория представлений алгебры $\widehat{L}$ эквивалентна категории пар $(M, \phi)$, где $M$ есть $L$-модуль, а $\phi: I \otimes M \rightarrow M-$ морфизм модулей, такой, что

$$
\phi \circ(\mathrm{id} \otimes \phi)((I \wedge I) \otimes M)=0,
$$




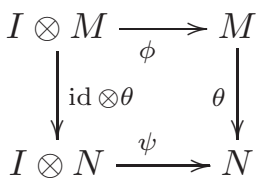

где $\theta$ - морфизм модулей. В далънейшем под парами мъ будем понимать паръ $(M, \phi)$.

Доказательство. Пусть $M$ есть $\widehat{L}$-модуль; тогда он является и $L$-модулем. Введем отображение $\phi: I \otimes M \rightarrow M: i \otimes m \mapsto i m$. Тогда для любых $i, j \in I, m \in M$

$$
\phi \circ(\mathrm{id} \otimes \phi)((i \otimes j-j \otimes i) \otimes(m))=i(j m)-j(i m)=[i, j] m=0 .
$$

Поэтому условие (1) выполняется.

Кроме того,

$$
l \phi(i \otimes m)=l i m=[l, i] m+i l m=\phi(l(i \otimes m)) .
$$

Поэтому $\phi-$ морфизм модулей.

Обратно, пусть $M$ есть $L$-модуль и $\phi: I \otimes M \rightarrow M-$ морфизм модулей, удовлетворяющий условию (1). Зададим действие элемента $i \in I$ на $m \in M$ по формуле $i m:=\phi(i \otimes m)$. В силу условия (1) получим, что $[i, j] m=i j m-j i m=0$ для $i, j \in I, m \in M$; кроме того, для $l \in L, i \in I, m \in M$

$$
[l, i] m=\phi([l, i] \otimes m)=\phi(l(i \otimes m)-i \otimes l m)=l \phi(i \otimes m)-i l m=l i m-i l m .
$$

Поэтому с таким действием $M$ является $\widehat{L}$-модулем.

Очевидно, что композиция двух описанных отображений является изоморфизмом. Тем самым задано соответствие на объектах категорий.

Пусть теперь $A: M \rightarrow N-$ морфизм $\widehat{L}$-модулей. Обозначим через $\theta$ тот же морфизм, рассматриваемый как морфизм $L$-модулей. Тогда для $i \in I, m \in M$

$$
\theta(\phi(i \otimes m))=\theta(i m)=A(i m)=i A(m)=i \theta(m)=\psi(i \otimes \theta(m)) .
$$

Это отображение $\theta$ определяет коммутативный квадрат (2). Далее, если два морфизма модулей делают коммутативным квадрат (2), то и их композиция тоже в силу функториальности тензорного произведения. Из тех же вычислений следует, что каждый коммутативный квадрат (2) задает отображение $\widehat{L}$-модулей и построенные в обе стороны функторы являются взаимно сопряженными. Тем самым эквивалентность рассматриваемых категорий доказана.

Замечание 3. Рассмотрим конечномерные представления (бесконечномерной) алгебры $L \wedge F(I)$, где $F(I)$ - свободная алгебра Ли, порожденная пространством $I$, причем $L$ действует на $I$ естественным образом, а ее действие на члены высших степеней определяется по правилу Лейбница. Тогда с помощью рассуждений, аналогичных доказательству предыдущей леммы, получаем, что категория конечномерных представлений алгебры $L \wedge F(I)$ эквивалентна категории пар $(M, \phi)$, где $M$ есть $L$-модуль, $\phi: I \otimes M \rightarrow M$, с морфизмами коммутативными квадратами (2) (но без условия (1)).

Зафиксируем теперь до конца параграфа полупростую алгебру Ли $L$ и $L$-модуль $I$. Занумеруем как-нибудь все попарно не эквивалентные неприводимые представления этой алгебры (это можно сделать, так как их счетное множество). Представление с номером $i$ будем обозначать через $M_{i}$. Введем теперь 
для алгебры Ли $L \curlywedge I$ счетный колчан $K_{I}$. Стрелок из вершины $k$ в вершину $l$ будет столько, какова кратность вхождения модуля $M_{l}$ в модуль $I \otimes M_{k}$. С этого момента и до конца параграфа буквы $\alpha$ и $\beta$ будут обозначать стрелки колчана $K_{I}$, а $\pi$ - пути длины 2. Для стрелки или пути $\mu$ обозначим через $s(\mu)$ и $t(\mu)$ начало и конец соответственно. Таким образом,

$$
I \otimes M_{k} \simeq \bigoplus_{k: s(\alpha)=k} M_{t(\alpha)}
$$

Следующая лемма является почти очевидной.

Лемма 2. Во введенных выше обозначениях

$$
I \otimes I \otimes M_{k} \simeq \bigoplus_{\pi: s(\pi)=k} M_{t(\pi)}
$$

Доказательство. Пользуясь формулой (3) два раза, получаем

$$
I \otimes I \otimes M_{k} \simeq \bigoplus_{s(\alpha)=k} I \otimes M_{t(\alpha)} \simeq \bigoplus_{s(\alpha)=k} \bigoplus_{s(\beta)=t(\alpha)} M_{t(\beta)} \simeq \bigoplus_{s(\pi)=k} M_{t(\pi)} .
$$

Лемма 3. (i) Категория конечномерных представлений колчана $K_{I}$ эквивалентна категории $\mathscr{K}_{I}$ пар $(M, \phi: I \otimes M \rightarrow M)$ с морфизмами - коммутативными квадратами (2).

(ii) Категория пар $(M, \phi: I \otimes M \rightarrow M)$ со свойством (1) эквивалентна категории конечномерных представлений колчана $K_{I}$ с однородными соотношениями степени 2 , причем размерность пространства соотношений на путях длины 2 из вершины $k$ в вершину $l$ равна кратности вхождения простого модуля $M_{l}$ в модуль $I \wedge I \otimes M_{k}$.

Доказательство. (i) Рассмотрим пару $p_{1}=(M, \phi: I \otimes M \rightarrow M)$. Разложим $M$ в сумму неприводимых компонент:

$$
M=\bigoplus_{i=1}^{\infty} V_{i} \otimes M_{i},
$$

где $V_{i}$ - конечномерное пространство и $\operatorname{dim}\left(V_{i}\right)$ - кратность вхождения модуля $M_{i}$ в $M$. При этом в силу конечномерности лишь конечное число пространств $V_{i}$ ненулевые. Тогда в силу формулы (3)

$$
I \otimes M \simeq \bigoplus_{i=1}^{\infty} V_{i} \otimes I \otimes M_{i} \simeq \bigoplus_{i=1}^{\infty} \bigoplus_{s(\alpha)=i} V_{i} \otimes M_{t(\alpha)}
$$

Следовательно, отображение $\phi$ распадается в прямую сумму отображений из $V_{i} \otimes M_{t(\alpha)}$ для всех $\alpha$, таких, что $s(\alpha)=i$ в $V_{j} \otimes M_{j}$. В силу леммы Шура для неизоморфных модулей $M_{t(\alpha)}$ и $M_{j}$ (т. е. в случае, когда $t(\alpha) \neq j$ ) существуют только нулевые морфизмы между $V_{i} \otimes M_{t(\alpha)}$ и $V_{j} \otimes M_{j}$, а для изоморфных морфизм имеет вид $A_{p_{1}, \alpha} \otimes \mathrm{id}$. Заметим, что построенный набор пространств $\left\{V_{i}\right\}$ и отображений $\left\{A_{p_{1}, \alpha}\right\}$ является представлением колчана $K_{I}$.

Рассмотрим морфизм пары $p_{1}=(M, \phi)$ в пару $p_{2}=(N, \psi)$, т. е. коммутативную диаграмму (2). Запишем разложения $M=\bigoplus_{i=1}^{\infty} V_{i} \otimes M_{i}, N=\bigoplus_{i=1}^{\infty} U_{i} \otimes M_{i}$. Морфизм $\theta$ распадается в прямую сумму морфизмов из $V_{i} \otimes M_{i}$ в $U_{j} \otimes M_{j}$, и для $i \neq j$ это отображение может быть только нулевым, а для $i=j$ оно имеет 
вид $T_{i} \otimes \mathrm{id}$, где $T_{i}$ - линейное отображение из $V_{i}$ в $U_{i}$. Морфизм id $\otimes \theta$ из диаграммы (2) тогда является прямой суммой морфизмов вида $T_{i} \otimes$ id из $V_{i} \otimes M_{t}(\alpha)$ в $U_{i} \otimes M_{t(\alpha)}$ для $s(\alpha)=i$. Запишем теперь условие коммутативности диаграммы (2) на каждом подмодуле $V_{s(\alpha)} \otimes M_{t(\alpha)}$ :

$$
T_{t(\alpha)} \otimes \mathrm{id} \circ A_{p_{1}, \alpha} \otimes \mathrm{id}=A_{p_{2}, \alpha} \otimes \mathrm{id} \circ T_{s(\alpha)} \otimes \mathrm{id},
$$

T. e.

$$
T_{t(\alpha)} \circ A_{p_{1}, \alpha}=A_{p_{2}, \alpha} \circ T_{s(\alpha)} .
$$

Но это - условие, что набор отображений $\left\{T_{i}\right\}$ является морфизмом представления $\left\{V_{i}, A_{p_{1}, \alpha}\right\}$ в $\left\{U_{i}, A_{p_{2}, \alpha}\right\}$. Тем самым мы получили отображение на объектах и морфизмах. Функториальность этого отображения очевидна.

Обратно, рассмотрим представление $\left\{V_{i}, A_{\alpha}\right\}$ колчана $K_{I}$. Построим $L$-модуль $M=\bigoplus_{i=1}^{\infty} V_{i} \otimes M_{i}$. Зададим морфизм $\phi: I \otimes M \rightarrow M$ набором отображений $A_{\alpha} \otimes \mathrm{id}: V_{s(\alpha)} \otimes M_{t(\alpha)} \rightarrow V_{t(\alpha)} \otimes M_{t(\alpha)}$. Тем самым мы задали отображение на объектах рассматриваемых категорий. Также по морфизму $\left\{T_{i}\right\}$ представлений колчана построим морфизм пар, задав набор отображений $T_{i} \otimes \mathrm{id}: V_{i} \otimes M_{i} \rightarrow$ $U_{i} \otimes M_{i}$. Как было показано выше, утверждение о том, что это действительно задает морфизм пар, эквивалентно тому, что $\left\{T_{i}\right\}$ - морфизм представлений колчана $K_{I}$. Тем самым мы построили функтор, и нетрудно видеть, что построенные здесь функторы являются взаимно сопряженными. Следовательно, рассматриваемые категории эквивалентны.

(ii) Рассмотрим полную подкатегорию пар $p=(M, \phi: I \otimes M \rightarrow M)$ со свойством (1). В силу формулы (6)

$$
I \otimes I \otimes M \simeq I \otimes \bigoplus_{i=1}^{\infty} \bigoplus_{s(\alpha)=i} V_{i} \otimes M_{t(\alpha)} \simeq I \otimes \bigoplus_{\alpha} V_{s(\alpha)} \otimes M_{t(\alpha)}
$$

Применив результаты п. (i), получаем, что образ прямого слагаемого $I \otimes$ $V_{s(\alpha)} \otimes M_{t(\alpha)}$ под действием отображения id $\otimes \phi$ будет лежать в $I \otimes V_{t(\alpha)} \otimes M_{t(\alpha)}$ и отображение на этом слагаемом будет иметь вид id $\otimes A_{p, \alpha} \otimes \mathrm{id}$. Следовательно,

$$
I \otimes V \otimes M_{t(\alpha)} \simeq V \otimes \bigoplus_{s(\beta)=t(\alpha)} M_{t(\beta)} .
$$

Поэтому $I \otimes V_{s(\alpha)} \otimes M_{t(\alpha)} \simeq \bigoplus_{t(\alpha)=s(\beta)} V_{s(\alpha)} \otimes M_{t(\beta)}$ и отображение id $\otimes \phi$ переводит $V_{s(\alpha)} \otimes M_{t(\beta)}$ в $V_{t(\alpha)} \otimes M_{t(\beta)}$ и имеет вид $A_{p, \alpha} \otimes \mathrm{id}$. Значит, отображение $\phi \circ(\mathrm{id} \otimes \phi)$ на каждом прямом слагаемом $V_{s(\alpha)} \otimes M_{t(\beta)}$ (напомним, что $t(\alpha)=s(\beta))$ имеет вид $A_{p, \beta} A_{p, \alpha} \otimes \mathrm{id}$.

Рассмотрим ограничение отображения $\phi \circ(\mathrm{id} \otimes \phi)$ на $(I \wedge I) \otimes M$, т. е. пару $p_{1}=(M, \phi \circ(\mathrm{id} \otimes \phi))$. На прямых слагаемых оно имеет вид $A_{p_{1}, \gamma} \otimes \mathrm{id}$, где $\gamma$ - стрелка в $K_{(I \wedge I)}$. Тогда условие $(1)$ эквивалентно тому, что все $A_{p_{1}, \gamma}$ равны нулю. Но по построению $A_{p_{1}, \gamma}$ являются линейными комбинациями линейных отображений вида $A_{p, \beta} A_{p, \alpha}$. Следовательно, условие (1) эквивалентно некоторому набору линейных соотношений на $A_{p, \beta} A_{p, \alpha}$, причем для вершин $k$ и $l$ количество соотношений для путей длины 2 равно кратности вхождения модуля $M_{l}$ в $(I \wedge I) \otimes M_{k}$. Поэтому эквивалентность категорий из п. (i) задает эквивалентность полных подкатегорий пар с условием (1) и представлений колчана $K_{I}$ с требуемым в условии леммы количеством соотношений длины 2 между каждой парой вершин. 
Теорема 1. Категория конечномерных представлений алгебры Ли $L \wedge F(I)$ эквивалентна категории представлений колчана $K_{I}$. Категория конечномерных представлений алгебры Ли $L \curlywedge I$ эквивалентна категории представлений колчана $K_{I}$ с набором соотношений второй степени, количество которых для путей из вершины $k$ в l равно кратности вхождения модуля $M_{l}$ в $(I \wedge I) \otimes M_{k}$.

Доказательство. Утверждение теоремы легко следует из леммы 3, замечания 3 и леммы 1.

\section{§4. Исследование представлений колчана $\boldsymbol{K}_{I}$, дикость алгебр Ли с абелевым радикалом}

Пусть $\mathbb{K} K_{I}$ - алгебра путей колчана $K_{I}$. Из теоремы 1 следует, что если для колчана $K_{I}$ алгебра $\mathbb{K} K_{I} / \operatorname{rad}\left(\mathbb{K} K_{I}\right)^{2}$ дикая, то и алгебра $L \wedge I$ дикая, так как категория ее представлений эквивалентна категории представлений алгебры с факторалгеброй, изоморфной алгебре $\mathbb{K} K_{I} / \operatorname{rad}\left(\mathbb{K} K_{I}\right)^{2}$. Очевидно, что алгебра $\mathbb{K} K_{I} / \operatorname{rad}\left(\mathbb{K} K_{I}\right)^{2}$ дикая, если $K_{I}$ - дикий колчан без последовательных стрелок. В общем случае имеется следующий, принадлежащий Габриелю критерий того, что алгебра $\mathbb{K} K_{I} / \operatorname{rad}\left(\mathbb{K} K_{I}\right)^{2}$ является дикой. Ссылку на него найти не удалось, так что приведем набросок доказательства.

Предложение 4. Пусть $Г-$ колчан. Тогда алгебра $\mathbb{K} \Gamma / \operatorname{rad}(\mathbb{K} \Gamma)^{2}$ является дикой в том и только том случае, когда следующий колчан $\Gamma^{\prime}$ является $\partial и-$ ким. Вершины колчана $\Gamma^{\prime}-$ это вершины $i^{\prime}, i^{\prime \prime}$ для каждой вершины $i$ из Г. Стрелок из $i^{\prime}$ в $j^{\prime \prime}$ столько же, сколько их из $i$ в $j$ в колчане $\Gamma$, и других стрелок нет.

Для доказательства нужно взять представление колчана Г, в качестве пространства в каждой вершине $j^{\prime \prime}$ взять сумму образов всех стрелок, входящих в $j$, а в качестве пространства в вершинах $j^{\prime}$ - фактор пространства в вершине $j$ по сумме этих образов. Таким образом мы получим все неразложимые представления колчана $\Gamma^{\prime}$ кроме тривиальных в вершинах $j^{\prime \prime}$. Колчан $\Gamma^{\prime}$ называется дублем Габриеля колчана Г.

Отсюда сразу же получаем следующее

Предложение 5. Пусть I есть L-модуль, для которого существует такой неприводимый $L$-модуль $M$, что $I \otimes M$ содержст 5 различных неприводимых компонент или компоненту кратности не менъше 3 , или компоненту кратности 2 и еще одну ненулевой кратности (и все эти компоненты отличны от $M)$. Тогда алгебра $L \wedge I$ дикал.

Доказательство. В описанных выше случаях дубль Габриеля колчана $K_{I}$ содержит один из следующих подколчанов:
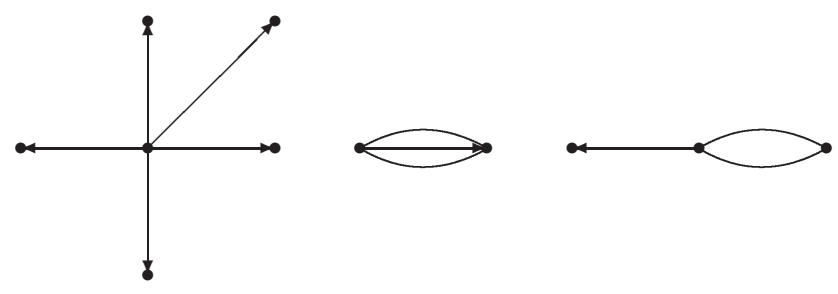

Эти колчаны дикие [8]. 
Предложение 6. Пусть $L$ - полупростая алгебра Ли, а I - простой $L$-модуль со старшим весом $\Lambda_{I}$, имеющий размерность больше 2. Тогда алгебра L人I дикая.

Доказательство. Если $\left\langle\Lambda_{N_{i}}, \alpha^{\vee}\right\rangle \geqslant 1, i=1,2$, для некоторых модулей $N_{1}$, $N_{2}$ со старшими весами $\Lambda_{N_{1}}, \Lambda_{N_{2}}$ и некоторого простого корня $\alpha$, то тензорное произведение $N_{1} \otimes N_{2}$ содержит простой модуль со старшим весом $\Lambda_{N_{1}}+\Lambda_{N_{2}}-\alpha$. Действительно, пусть $n_{1}, n_{2}$ - старшие векторы этих модулей. Рассмотрим вектор $\left\langle\Lambda_{N_{2}}, \alpha^{\vee}\right\rangle f_{\alpha} n_{1} \otimes n_{2}-\left\langle\Lambda_{N_{1}}, \alpha^{\vee}\right\rangle n_{1} \otimes f_{\alpha} n_{2}$. Нетрудно видеть, что этот элемент имеет вес $\Lambda_{N_{1}}+\Lambda_{N_{2}}-\alpha$, является ненулевым, так как $f_{\alpha} n_{i}=0$ тогда и только тогда, когда $\left\langle\Lambda_{N_{i}}, \alpha^{\vee}\right\rangle=0$, и аннулируется всеми положительными корневыми векторами. Следовательно, он является старшим весовым элементом некоторого простого подмодуля.

Если же $\left\langle\Lambda_{N_{i}}, \alpha^{\vee}\right\rangle \geqslant 2$, то все элементы $f_{\alpha} f_{\alpha} n_{1} \otimes n_{2}, f_{\alpha} n_{1} \otimes f_{\alpha} n_{2}, n_{1} \otimes f_{\alpha} f_{\alpha} n_{2}$ ненулевые. Если $\beta$ - простой корень, $\beta \neq \alpha$, то $e_{\beta}$ аннулирует все эти элементы, так как коммутирует с $f_{\alpha}$. Элемент $e_{\alpha}$ переводит пространство, порожденное этими элементами, в двумерное. Поэтому существует линейная комбинация элементов $f_{\alpha} f_{\alpha} n_{1} \otimes n_{2}, f_{\alpha} n_{1} \otimes f_{\alpha} n_{2}, n_{1} \otimes f_{\alpha} f_{\alpha} n_{2}$, аннулируемая элементом $e_{\alpha}$. Но тогда эта линейная комбинация аннулируется всеми положительными корневыми векторами, т. е. является старшим весом некоторого простого подмодуля. Поэтому тензорное произведение $N_{1} \otimes N_{2}$ содержит простой подмодуль со старшим весом $\Lambda_{N_{1}}+\Lambda_{N_{2}}-2 \alpha$.

(i) Рассмотрим простой модуль $M$, такой, что для его старшего веса $\Lambda_{M}$ формы $\Lambda_{M}+\alpha, \Lambda_{M}+\beta$ также являются доминантными и $\left\langle\Lambda_{M}, \alpha^{\vee}\right\rangle \geqslant 1$, $\left\langle\Lambda_{M}, \beta^{\vee}\right\rangle \geqslant 1$. По доказанному выше тензорное произведение $I \otimes M$ содержит подмодули со старшими весами $\Lambda_{M}+\Lambda_{I}-\alpha, \Lambda_{M}+\Lambda_{I}-\beta$. Также это произведение содержит простой подмодуль со старшим весом $\Lambda_{M}+\Lambda_{I}$. Далее, для модуля $N$ со старшим весом $\Lambda_{M}+\alpha$ в модуле $I \otimes N$ есть подмодули со старшими весами $\Lambda_{M}+\Lambda_{I}, \Lambda_{M}+\Lambda_{I}+\alpha$, и для модуля со старшим весом $\Lambda_{M}+\beta$ один из простых подмодулей тензорного произведения этого модуля на $I$ имеет старший вес $\Lambda_{M}+\Lambda_{I}$ (так как $\left.\left\langle\Lambda_{M}+\alpha, \alpha^{\vee}\right\rangle \geqslant 1,\left\langle\Lambda_{M}+\beta, \beta^{\vee}\right\rangle \geqslant 1\right)$. Все эти модули различны в силу свойств рассматриваемого модуля $I$, кроме случая $\Lambda_{I}=\alpha+\beta$. Поэтому колчан $K_{I}$ содержит следующий подколчан:

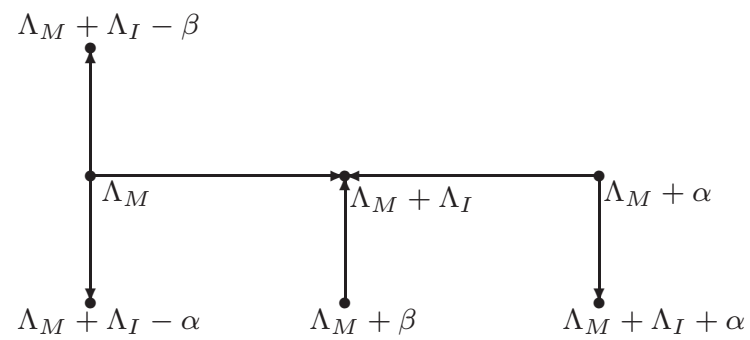

В случае $\Lambda_{I}=\alpha+\beta$ дубль Габриеля колчана $K_{I}$ будет содержать такой подколчан.

Этот колчан дикий и без последовательных стрелок; поэтому в силу теоремы 1 алгебра $L \curlywedge I$ дикая.

(ii) Пусть $M$ - такой простой модуль, что для его старшего веса $\Lambda_{M}$ форма $\Lambda_{M}-2 \alpha$ также является доминантной. Тогда $\left\langle\Lambda_{M}-\alpha, \alpha^{\vee}\right\rangle \geqslant 1 ;$ следовательно, 
по доказанному выше $I \otimes M$ содержит простые модули весов $\Lambda_{M}+\Lambda_{I}, \Lambda_{M}+$ $\Lambda_{I}-\alpha, \Lambda_{M}+\Lambda_{I}-2 \alpha$, а произведение модуля со старшим весом $\Lambda_{M}-\alpha$ на $I$ содержит подмодули со старшими весами $\Lambda_{M}+\Lambda_{I}-\alpha, \Lambda_{M}+\Lambda_{I}-2 \alpha$. Поэтому в зависимости от того, равняется ли $\Lambda_{I}$ одному из весов $\alpha$ или $2 \alpha$, или ни тому, ни другому, $K_{I}$ содержит один из следующих подколчанов:
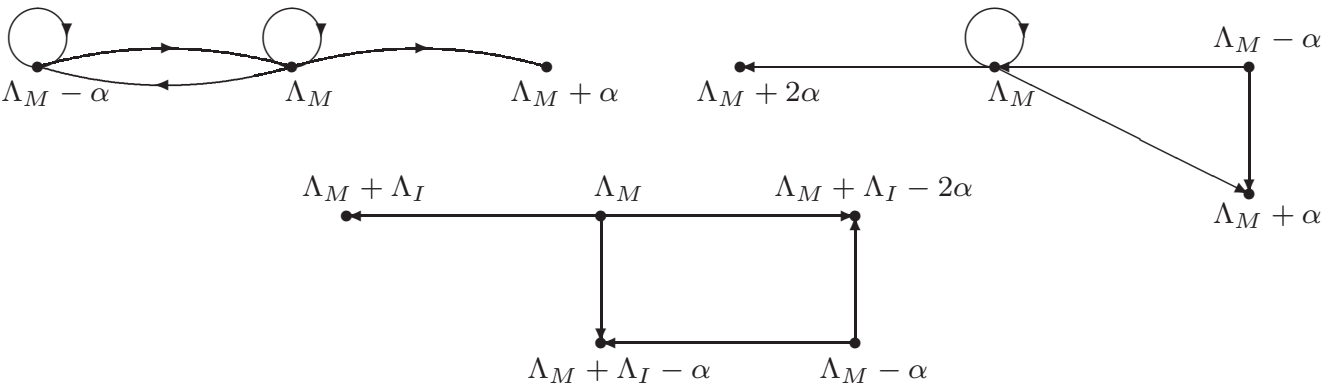

$\Lambda_{M}+\alpha$

У всех этих колчанов является диким фактор алгебры путей по квадрату радикала (так как диким является дубль Габриеля). Поэтому алгебра $L \wedge I$ дикая.

(iii) Из доказанного выше следует, что алгебра Ли $L \curlywedge I$, где $L$ полупроста, a $I$ есть $L$-модуль, является дикой, если в $I$ присутствует нефундаментальное представление алгебры $L$. Напомним, что представление называется фундаментальным, если его старший вес равен нулю на всех элементах $h_{\alpha}$ для простых корней $\alpha$, кроме одного, на котором он равен 1.

Рассмотрим фундаментальное представление модуля $I$. Пусть $m$ - его старший весовой вектор. Пусть $\alpha$ - такой (единственный) простой корень, что $f_{\alpha} m \neq 0$. Если это представление не двумерно, то существует такой простой корень $\beta$, что $f_{\beta} f_{\alpha} m \neq 0$. Пусть теперь $N$ - такой $L$-модуль, что для его старшего весового вектора $n$ элементы $f_{\alpha} f_{\beta} n$ и $f_{\beta} f_{\alpha} n$ линейно независимы и то же самое верно для модуля со старшим весом $\Lambda_{N}+\alpha$. Рассмотрим элементы $f_{\alpha} f_{\beta} m \otimes n, f_{\beta} m \otimes f_{\alpha} n, m \otimes f_{\alpha} f_{\beta} n, m \otimes f_{\beta} f_{\alpha} n$ веса $\Lambda_{N}+\Lambda_{I}-\alpha-\beta$ тензорного произведения $I \otimes N$. Они линейно независимы. Непосредственным вычислением получаем, что оператор умножения на $e_{\alpha}$ имеет на их линейной оболочке одномерный образ, а оператор умножения на $e_{\beta}$ - двумерный. Поэтому ядра этих операторов имеют ненулевое пересечение, а следовательно, существует ненулевой элемент веса $\Lambda_{N}+\Lambda_{I}-\alpha-\beta$, аннулируемый всеми положительными корневыми векторами. Поэтому в рассматриваемом тензорном произведении существует простой подмодуль со старшим весом $\Lambda_{N}+\Lambda_{I}-\alpha-\beta$. Значит, аналогично тому, как это сделано в предыдущем пункте, получаем в колчане $K_{I}$ один из описанных в этом пункте подколчанов. Следовательно, если модуль $I$ простой и имеет размерность больше 2 , то алгебра $L<I$ дикая.

Если модуль $I$ не является простым, $I=J \oplus J^{\prime}$, то алгебра $L \ltimes J$ является факторалгеброй алгебры $L \wedge I$. Поэтому из предложения 6 следует, что алгебра $L \nless I$ дикая, если одно из неприводимых прямых слагаемых модуля $I$ имеет размерность больше двух.

\section{1. Случай двумерного модуля.}

Лемма 4. Алгебры Ли с двумерным абелевым радикалом дикие. 
Доказательство. Если радикал приводим как модуль над полупростой частью, т. е. распадается в прямую сумму двух одномерных, то колчан $K_{I}$ представляет собой несвязное объединение вершин с двумя петлями, так как тензорное произведение любого модуля $N$ на одномерный изоморфно $N$. Модуль $I \wedge I$ одномерен, поэтому получаем задачу описания представлений пары матриц с одним однородным соотношением второй степени. Эта задача дикая при любом соотношении в силу работы [6]. Остается случай двумерного неприводимого модуля. Двумерный неприводимый модуль $I$ бывает только над алгебрами Ли вида $L=s l_{2} \oplus \widetilde{L}$, где $\widetilde{L}$ - полупростая алгебра, действующая на $I$ тривиально. В этом случае $L \wedge I \simeq s l_{2} \wedge I \oplus \widetilde{L}$. Вследствие предложения 3 прямая сумма полупростой и ручной алгебр Ли ручная. Поэтому достаточно исследовать алгебру $s l_{2} \curlywedge I$.

Рассмотрим случай двумерного модуля над $s l_{2}$. В силу формулы КлебшаГордона колчан $K_{I}$ для такого модуля имеет вид

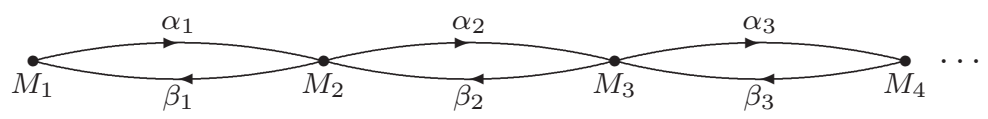

Модуль $I \wedge I$ изоморфен одномерному модулю. Поэтому все соотношения будут иметь вид $k_{i} \alpha_{i} \beta_{i}+l_{i} \beta_{i+1} \alpha_{i+1}=0$ при некоторых константах $k_{i}$ и $l_{i}$. Эта задача дикая при любых константах, так как у подколчана на его шести последовательных вершинах имеется накрывающая алгебра, у которой форма Титса не является неотрицательно определенной.

Форма Титса для колчана с соотношениями определяется на пространстве $\mathbb{K}^{n}$, где $n$ - число вершин колчана, следующим образом. Пусть $\left\{v_{1}, \ldots, v_{n}\right\}-$ базис пространства $\mathbb{K}^{n},\left\{e_{1}, \ldots, e_{n}\right\}$ - множество вершин колчана, $l_{i j}$ - число стрелок из $e_{i}$ в $e_{j}, r_{i j}$ - размерность пространства соотношений на пути из $e_{i}$ в $e_{j}$. Тогда форма Титса - это следующая квадратичная форма:

$$
T\left(\sum_{i=1}^{n} \alpha_{i} v_{i}\right)=\sum_{i=1}^{n} \alpha_{i}^{2}-\sum_{i, j=1}^{n} \frac{1}{2}\left(l_{i j}+l_{j i}\right) \alpha_{i} \alpha_{j}+\sum_{i, j=1}^{n} \frac{1}{2}\left(r_{i j}+r_{j i}\right) \alpha_{i} \alpha_{j} .
$$

Искомая накрывающая алгебра - это алгебра путей колчана $Q$

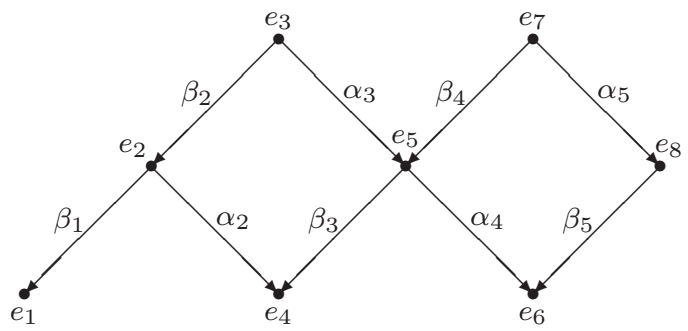

с одним линейным соотношением на $\beta_{2} \alpha_{2}, \alpha_{3} \beta_{3}$ и одним соотношением на $\beta_{4} \alpha_{4}$, $\alpha_{5} \beta_{5}$, а именно $k_{2} \alpha_{2} \beta_{2}+l_{2} \beta_{3} \alpha_{3}=0$ и $k_{4} \alpha_{4} \beta_{4}+l_{4} \beta_{5} \alpha_{5}=0$. Форма Титса этого колчана принимает отрицательное значение на векторе $v_{1}+2 v_{2}+2 v_{3}+2 v_{4}+$ $4 v_{5}+2 v_{6}+2 v_{7}+2 v_{8}$.

Опишем функтор из категории представлений рассматриваемого колчана $Q$ в категорию представлений подколчана колчана $K_{I}$, содержащего вершины, 
соответствующие модулям размерности от 1 до 6, и все стрелки между этими вершинами. Пусть $U$ - некоторое представление колчана $Q, U_{i}$ - пространства, отвечающие вершинам, $U_{\alpha}$ - отображения между ними. Положим $V_{1}=U_{1}$, $V_{2}=U_{2}, V_{3}=U_{3} \oplus U_{4}, V_{4}=U_{5}, V_{5}=U_{6} \oplus U_{7}, V_{6}=U_{8}$. Далее, положим $V_{\alpha_{1}}=0$, $V_{\beta_{1}}=U_{\beta_{1}}, V_{\alpha_{2}}=\left(0, U_{\alpha_{2}}\right), V_{\beta_{2}}=\left(0, U_{\beta_{2}}\right)^{t}, V_{\alpha_{3}}=\left(U_{\alpha_{3}}, 0\right), V_{\beta_{3}}=\left(U_{\beta_{3}}, 0\right)^{t}, V_{\alpha_{4}}=$ $\left(0, U_{\alpha_{4}}\right), V_{\beta_{4}}=\left(0, U_{\beta_{4}}\right)^{t}, V_{\alpha_{5}}=\left(U_{\alpha_{5}}, 0\right), V_{\beta_{5}}=\left(U_{\beta_{5}}, 0\right)^{t}$, где запись $(a, b)$ означает отображение $a$ в первое прямое слагаемое и отображение $b$ во второе, а запись $(a, b)^{t}$ означает сумму отображений из первого и второго прямых слагаемых.

Непосредственно проверяется, что построенное отображение действительно задает функтор из категории представлений одного колчана в категорию представлений другого, и тот факт, что этот функтор переводит неразложимые представления в неразложимые и неизоморфные в неизоморфные.

Поэтому алгебра $s l_{2} \curlywedge I$ дикая.

Следовательно, можно доказать такую теорему.

Теорема 2. Пусть $\widehat{L}=L \wedge I-$ алгебра Ли с абелевым радикалом. Тогда она является ручной в том и только том случае, когда модуль I одномерен. В противном случае $\widehat{L}$ дикая.

Доказательство. Если размерность модуля $I$ больше единицы, то $\widehat{L}$ является дикой по лемме 4 или предложению 6.

\section{§5. Случай неабелева радикала}

Рассмотрим теперь алгебру Ли $L$ с неабелевым радикалом $R$. Алгебра $L /[R, R]$ имеет абелев радикал. Если она дикая, то и $L$ дикая. Все алгебры $L /[R, R]$, являющиеся ручными, описываются теоремой 2 .

5.1. Алгебры с одномерным фактором радикала по его коммутанту. Теперь рассмотрим случай, когда фактор радикала по его коммутанту одномерен. Будем считать алгебру $L$ неразрешимой. Очевидно, что чтобы доказать дикость таких алгебр, достаточно доказать дикость их факторов по второму коммутанту радикала. Поэтому будем считать, что коммутант радикала абелев.

Рассмотрим расширения при помощи модуля $J$ алгебр вида $L_{0} \oplus I$, где $L_{0}$ полупроста, а $I$ одномерна. Очевидно, что модуль $J$ можно считать неприводимым (если это не так, можно перейти к факторалгебре), т. е. в силу предложения 3 просто $L_{0}$-модулем, на котором некоторая образующая алгебры $I$ действует тождественным либо нулевым образом. Во втором случае получаем алгебру с абелевым радикалом размерности больше чем 1. Поэтому все такие алгебры дикие в силу теоремы 2. Следовательно, достаточно рассмотреть случай, когда $J$ является неприводимым $L_{0}$-модулем и некоторая образующая алгебры $I$ действует на этом модуле тождественно. Кроме того, расширение можно считать полупрямым произведением, так как алгебра $L_{0} \oplus I$ имеет тривиальные вторые когомологии (см, например, [10]). Теперь достаточно доказать следующее предложение.

Предложение 7. Пусть $L=\left(L_{0} \oplus I\right) \wedge J$, где алгебра $L_{0}$ nолупроста, $I$

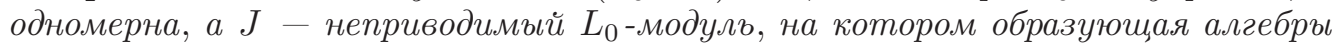
I действует тождественно. Тогда алгебра L дикая. 


\section{Доказательство. Рассмотрим колчан $Q$}

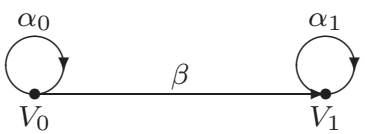

с соотношением $\alpha_{1} \beta=\beta \alpha_{0}+\beta$.

Этот колчан дикий (см., например, [5]). Построим функтор из категории представлений этого колчана в категорию представлений алгебры $L=$ $\left(L_{0} \oplus I\right) \wedge J$. Пусть $\left(V_{0}, V_{1}, \alpha_{0}, \alpha_{1}, \beta\right)$ - представление рассматриваемого колчана, где $V_{0}, V_{1}$ - пространства в вершинах, $\alpha_{i}: V_{i} \rightarrow V_{i}, \beta: V_{0} \rightarrow V_{1}$. Построим следующее представление алгебры $L$. Пусть $M=V_{0} \oplus V_{1} \otimes J$ как $L_{0}$-модули. Выберем образующую $e$ в алгебре $I, I=\langle e\rangle$, и положим $e \cdot v_{0}=\alpha_{i}\left(v_{0}\right)$, $e \cdot v_{1} \otimes j=\alpha_{1}\left(v_{1}\right) \otimes j, j \cdot v_{0}=\beta\left(v_{0}\right) \otimes j, j \cdot v_{1} \otimes j^{\prime}=0$ для $v_{k} \in V_{k}$ и $j, j^{\prime} \in J$. Легко проверяется, что это представление и что естественно определенное отображение на морфизмах задает точный функтор $F$. Предположим, что построенное представление разложимо. Пусть элемент $v_{0}+v_{1} \otimes j$ принадлежит одному прямому слагаемому, $v_{0}+v_{1} \otimes j \in U^{\prime}$. Тогда умножением на элементы из полупростой алгебры $L_{0}$ мы получим, что $v_{1} \otimes J \subseteq U^{\prime}$, в частности, $v_{1} \otimes j \in U^{\prime}$, а значит, и $v_{0} \in U^{\prime}$. Поэтому прямые слагаемые имеют вид $U_{0}^{\prime} \oplus U_{1}^{\prime} \otimes J, U_{0}^{\prime \prime} \oplus U_{1}^{\prime \prime} \otimes J$. Очевидным образом получаем, что пространства $U_{0}^{\prime}, U_{0}^{\prime \prime}$ инвариантны под действием $\alpha_{0}$, а $U_{1}^{\prime}, U_{1}^{\prime \prime}$ - под действием $\alpha_{1}$ и $\beta$ переводит $U_{0}^{\prime}$ в $U_{1}^{\prime}$ и $U_{0}^{\prime \prime}$ в $U_{1}^{\prime \prime}$. Поэтому мы получили разложение представления колчана. Следовательно, построенный функтор переводит неразложимые представления в неразложимые.

Рассмотрим произвольный морфизм $\theta: U_{0} \oplus U_{1} \otimes J \rightarrow V_{0} \oplus V_{1} \otimes J L$-модулей рассматриваемого вида. Тогда в силу леммы Шура получаем, что $\theta\left(U_{0}\right) \subseteq V_{0}$, $\theta\left(U_{1} \otimes J\right) \subseteq V_{1} \otimes J$, причем $\theta\left(U_{1} \otimes J\right)=\eta\left(U_{1}\right) \otimes J$ для некоторого линейного оператора $\eta$. Непосредственно проверяется, что $\left.\theta\right|_{U_{0}}$ и $\eta$ задают представление рассматриваемого колчана с соотношениями. Построенное отображение функториально и является сопряженным функтором к построенному выше, если рассматривать его как функтор на полную подкатегорию модулей вида $U \oplus U \otimes J$. Поэтому построенный нами функтор является вложением категорий.

Из этого следует, что все алгебры Ли вида $L=\left(L_{0} \oplus I\right) \wedge J$ дикие.

Замечание 4. Из результатов этого параграфа и теоремы 2 следует, что все алгебры Ли с неабелевым радикалом дикие.

5.2. Основная теорема. Из всего доказанного выше вытекает следующая теорема:

Теорема 3. Ручными являются следующие алгебры Ли:

(1) полупростые;

(2) прямые суммы полупростой и одномерной.

Все остальные алгебры Ли дикие.

Доказательство. Все полупростые алгебры Ли ручные в силу классической теории представлений полупростых алгебр Ли. Все разрешимые алгебры являются дикими в силу предложения 2.

Будем теперь считать, что данная алгебра Ли не содержит разрешимых прямых слагаемых. 
Рассмотрим разложение Леви алгебры Ли $\widehat{L}: \widehat{L}=L \curlywedge R$, где $L$ полупроста, а $R$ - радикал. Если $R /[R, R]$ одномерна, то в силу предложений 7 и $3 \widehat{L}$ является дикой тогда и только тогда, когда $R$ не одномерен, и ручной, когда $R$ одномерен.

Пусть теперь $\operatorname{dim} R /[R, R]>1$. Тогда $\widehat{L} /[R, R]$ является дикой в силу теоремы 2. Поэтому $\widehat{L}$ тоже дикая.

Замечание 5. В работе [10] приведен список конечномерных алгебр Ли, имеющих тривиальные вторые когомологии в любом конечномерном модуле. Этот список совпадает со списком ручных алгебр Ли. Таким образом, конечномерная алгебра Ли является ручной тогда и только тогда, когда она имеет тривиальные вторые когомологии со значениями в любом конечномерном модуле.

Автор выражает благодарность профессору Ю. С. Самойленко за постановку задачи и помощь при написании работы, профессору С. А. Овсиенко за помощь в некоторых доказательствах и рецензенту за ценные замечания.

\section{ЛитеРАТУРА}

[1] И. М. Гельфанд, В. А. Пономарев, Замечания о классификации пары коммутирующих линейных преобразований в конечномерном пространстве, Функц. анализ и его прил., 3:4 (1969), 81-82.

[2] Ю. А. Дрозд, Ручные и дикие матричные задачи, в кн.: Матричные задачи, АН УкрССР, Институт математики, Киев, 1977, 104-114.

[3] Ю. А. Дрозд, Представления коммутативных алгебр, Функц. анализ и его прил., 6:4 (1973), 41-43.

[4] Yu. A. Drozd, G.-M. Greuel, I. Kashuba, On Cohen-Macaulay modules on surface singularities, Mosc. Math. J., 3:2 (2003), 397-418.

[5] Yang Han, Wild two-point algebras, J. Algebra, 247:1 (2002), 57-77.

[6] В. Л. Островский, Ю. С. Самойленко, О парах операторов, связанных квадратичным соотношением, Функц. анализ и его прил., 47:1 (2013), 82-87.

[7] М. Гото, Ф. Гроссханс, Полупростые алгебры Ли, Мир, М., 1981.

[8] Л. А. Назарова, Представления колчанов бесконечного типа, Изв. АН СССР, $\mathbf{3 7 : 4}$ (1973), 752-791.

[9] P. Gabriel, A. V. Roiter, Representations of Finite Dimensional Algebras, Encuclopaedia of Math. Sci., vol. 73 (Algebra 8), Springer-Verlag, Berlin, 1992.

[10] P. Zusmanovich, A converse to the second Whitehead Lemma, J. Lie Theory, 18:2 (2008), 295-299.

[11] K. Morita, Duality of modules and its applications to the theory of rings with minimal condition, Sci. Rep. Tokyo Kyoiku Diagaku. Sect. A, 6 (1958), 83-142.

Механико-математический факультет,

КНУ им. Т. Г. Шевченко, Киев

e-mail: makedonskyi@univ.kiev.ua
Поступило в редакцию 14 декабря 2011 г. 